\title{
REVISTA CHILENA DE DERECHO Y TECNOLOGÍA
}

SEGUNDO SEMESTRE 2015 VOL. 4 NÚM. 2 
REPRESENTANTE LEGAL

PROF. DAVOR HARASIC YAKSIC

Decano, Facultad de Derecho, Universidad de Chile.

DIRECTOR RESPONSABLE

PROF. ALEX PESSÓ STOULMAN

EDITOR GENERAL

PROF. DANIEL ÁLVAREZ VALENZUELA

\section{COMITÉ EDITORIAL}

MG. ALBERTO CERDA

Profesor Asistente, Facultad de Derecho, Universidad de Chile, Chile. LL.M. in International Legal Studies, Georgetown University. Magister en Derecho Público, Universidad de Chile.

\section{MG. MARCELO CORRALES}

Investigador Senior, Institute for Legal Informatics, Leibniz University of Hanover, Alemania. LL.M. in European Intellectual Property Law,

Stockholm University. LL.M. in Law and Information Technology, Stockholm University.

DR. CARLOS DELPIAZZO

Profesor de Derecho Administrativo y Derecho Informático, Universidad de la República, Uruguay. Doctor en Derecho y Ciencias Sociales, Universidad de la República, Uruguay.

DR. RONALDO LEMOS

Director, Instituto de Tecnologia \& Sociedade do Rio de Janeiro. Doctor en Derecho, Universidad de Sao Paulo, Brasil. LL.M. in Law, Harvard University.

DR. JULIO TÉLLEZ

Investigador Titular Derecho de la Informática. Universidad Nacional de México, México. Doctor en Informática Jurídica y Derecho de la informática, Universidad de Montpellier I, Francia. 
La Revista Chilena de Derecho y Tecnología es una publicación semestral del Centro de Estudios en Derecho Informático de la Facultad de Derecho de la Universidad de Chile que tiene por objeto difundir en la comunidad jurídica nacional, regional e internacional, el conocimiento científico relevante y necesario para analizar y comprender los alcances y efectos que el desarrollo tecnológico y cultural han producido en la sociedad, especialmente su impacto en las ciencias jurídicas y sociales.

\section{Revista Chilena de Derecho y Tecnología}

Rev. chil. derecho tecnol. (impr.)

Centro de Estudios en Derecho Informático

Facultad de Derecho $\cdot$ Universidad de Chile

Pío Nono núm. I, $4 \cdot^{\circ}$ piso, Providencia

Santiago de Chile

$+56229785263$

rchdt@derecho.uchile.cl

http://www.cedi.uchile.cl

http://twitter.com/rchdt

ISSN 07I9-2584

La Revista Chilena de Derecho y Tecnología es publicada en formatos electrónicos (pdf, epub y mobi) disponibles para descarga en la página web $<$ http://www.rchdt.uchile.cl/>.

Una guía para la presentación de manuscritos a la Revista Chilena de Derecho $y$ Tecnología está disponible en el siguiente enlace: <http://www.revistas. uchile.cl/index.php/rchdt/about/submissions\#authorguidelines>.

Algunos derechos reservados.

Publicada bajo los términos de la licencia Creative Commons ATRIBUCIÓN - NO COMERCIAL - COMPARTIR IGUAL 2.0 CHILE.

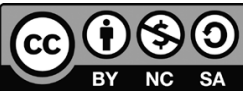




\section{REVISTA CHILENA DE DERECHO Y TECNOLOGÍA SEGUNDO SEMESTRE 2015 VOL. 4 NÚM. 2 Rev. chil. derecho tecnol. (impr.)}

7-8 Editorial

9-5I PAULA JERVIS ORTIZ

Internet de las cosas y protección de datos personales

53-74 ARTURO J. CARRILLO Y DAWN C. NUNZIATO

El precio de la priorización pagada: Las consecuencias internacionales y domésticas del fracaso de proteger la neutralidad en la red en los Estados Unidos

75-IO7 FELIPE FIGUEROA ZIMMERMANN

¿Qué significa justificar el derecho de autor?

IO9-I77 FERDINAND SCHNETTLER CHÁVEZ

Responsabilidad civil extracontractual de los usuarios de programas P2P por infracción a los derechos de autor

I79-2I3 MARÍA PAZ CANALES LOEBEL Oracle versus Google: Protección de derecho de autor sobre elementos funcionales de programas computacionales que ponen en riesgo la interoperabilidad y la innovación

2I5-26I ANA MARÍA MUÑOZ MASSOUH

Eliminación de datos personales en internet:

El reconocimiento del derecho al olvido 


\title{
El precio de la priorización pagada: Las consecuencias internacionales y domésticas del fracaso de proteger la neutralidad en la red en los Estados Unidos
}

\author{
The Price of Paid Prioritization. \\ The International and Domestic Consequences of the Failure \\ to Protect Net Neutrality in the United States
}

\author{
ARTURO J. CARRILLO Y DAWN C. NUNZIATO \\ George Washington University Law School, Estados Unidos
}

RESUMEN En este artículo examinamos, en primer lugar, las obligaciones en el ámbito internacional sobre comercio y derechos humanos de Estados Unidos en relación con la neutralidad en la red, para determinar hasta qué punto las dos últimas aproximaciones adoptadas por la FCC para promover una internet libre y abierta cumplen con dichas obligaciones. También examinamos la historia de la regulación de los proveedores de banda ancha como common carriers sujetos a obligaciones de no discriminación bajo la ley estadounidense. Concluimos que la nueva "Open Internet Order» de la FCC cumple, en términos generales, de forma exitosa las obligaciones internacionales de comercio y derechos humanos contraídas por Estados Unidos.

PALABRAS CLAVE Neutralidad de internet, Orden de Internet Abierta, FCC, derecho internacional, derechos humanos. 


\begin{abstract}
In this article, we first examine the trade and human rights obligations of the United States as they relate to net neutrality to determine the extent to which each of the two most recent approaches adopted by the FCC to promote an open Internet would comply with those obligations. We also examine the history of regulation of broadband providers as common carriers subject to nondiscrimination obligations under U.S. law. We conclude that the FCC has, by and large, successfully complied with its international trade and human rights obligations in its new Open Internet Order.
\end{abstract}

KEYWORDS Net neutrality, Open Internet Order, FCC, international law, human rights.

\title{
INTRODUCCIÓN
}

El Io de noviembre de 20I4, el presidente Barack Obama reafirmó su compromiso con una internet libre y abierta y llamó a la Federal Communications Commission (FCC) a «implementar las reglas más estrictas posibles para proteger la neutralidad en la red». ${ }^{\mathrm{I}}$ En particular, el presidente recomendó que la FCC reclasificara a los proveedores de ban$\mathrm{da}$ ancha como servicios de telecomunicaciones sujetos a obligaciones de common carriage. ${ }^{2}$ El presidente estaba respondiendo a la sección «No Commercially Unreasonable Practices» de las Proposed Rules for Promoting and Protecting an Open Internet (en adelante, las «Reglas Propuestas») de mayo de 20I4, las que habrían autorizado a los proveedores de banda ancha a concertar un trato diferenciado en el tráfico de internet, socavando de esta forma la neutralidad en la red y los principios comunes de common carriage en los Estados Unidos.

Como estaban redactadas, las Reglas Propuestas habrían violado obligaciones adquiridas por Estados Unidos en distintos tratados inter-

I. The White House, «Net Neutrality: President Obama's Plan for a Free and Open Internet» (20I4), disponible en <http://www.whitehouse.gov/net-neutrality>.

2. En el derecho anglosajón, common carrier es quien provee servicios de transporte de un determinado bien o personas, previa licencia, concesión o autorización del organismo regulador, sin discriminación y haciéndose responsable por las pérdidas. En términos específicos, se refiere a las empresas que prestan servicios públicos de telecomunicaciones. (N. del T.) 
nacionales de comercio y derechos humanos. Esto porque, como miembro de la Organización Mundial del Comercio (OMC) y parte del Pacto Internacional de Derechos Civiles y Políticos (el Pacto Internacional o PIDCP), Estados Unidos está obligado a respetar los principios de no discriminación y libertad de expresión al momento de regular medios de comunicación esenciales como internet. Cualquier regulación de la FCC que no proteja sustantivamente la neutralidad en la red en todos los niveles de interconectividad, infringiría estas obligaciones legales y expondría a Estados Unidos a acciones legales de otros gobiernos e individuos perjudicados por dicha regulación.

El I 2 de marzo de 2015 , la FCC adoptó un nuevo conjunto de reglas para promover y proteger una internet abierta. ${ }^{3}$ En su Open Internet Order de 2015 , la FCC reclasifica a los proveedores de banda ancha como common carriers sujetos a obligaciones de no discriminación y consagra importantes protecciones a la neutralidad en la red. La Open Internet Order de 2015 , contraria a su predecesora, cumple ampliamente los requerimientos de los tratados internacionales de comercio y derechos humanos de los cuales Estados Unidos es parte. Aun así, algunas lagunas en las nuevas reglas implican que Estados Unidos todavía puede resultar responsable ante el derecho internacional en caso de no poder asegurar que la neutralidad en la red y el trato no discriminatorio se encuentren adecuadamente protegidos.

En este artículo examinamos primeramente las obligaciones del derecho internacional del comercio y de los derechos humanos de Estados Unidos en relación con la neutralidad en la red, para determinar hasta qué punto las dos últimas aproximaciones adoptadas por la FCC para promover una internet libre y abierta cumplen con dichas obligaciones. También examinamos la historia de la regulación de los proveedores de banda ancha como common carriers sujetos a obligaciones de no discri-

3. El I 2 de marzo de 20 I 5 , la FCC liberó la última versión del Open Internet Order, detallando nuevas y potentes reglas de neutralidad en la red. Véase Open Internet Order (2015). Véase también Somer (2015), que discute como las reglas de 2015 superaron las expectativas al prohibir el bloqueo, la degradación y la priorización pagada, tanto para banda ancha móvil como fija; el Center for Democracy and Technology (2013: 4-9) detalla cómo la neutralidad en la red implica obligaciones de derechos humanos concernientes a la libertad de expresión y la no discriminación. 
minación bajo la ley estadounidense. Concluimos que la Open Internet Order de 2015 de la FCC cumple, en términos generales, de forma exitosa las obligaciones internacionales de comercio y derechos humanos contraídas por Estados Unidos.

\section{CONSECUENCIAS PARA EL COMERCIO INTERNACIONAL DEL FRACASO DE LA FCC DE ADOPTAR REGLAS SUSTANTIVAS PARA PROTEGER LA NEUTRALIDAD EN LA RED}

Estados Unidos se encuentra obligado por el Acuerdo General sobre el Comercio de Servicios (GATS por su nombre en inglés) de la OMC, y, adicionalmente, por el Acuerdo Básico de Telecomunicaciones (BATS por su nombre en inglés), a regular sus servicios de telecomunicación sobre la base de distintos principios que son esenciales para la neutralidad en la red. ${ }^{4}$ En particular, el BATS consagra el compromiso de Estados Unidos de asegurar que la «interconexión» en servicios telecomunicacionales, incluyendo el servicio de internet, sea otorgada a los proveedores de servicios de otros Estados parte de la OMC en términos no discriminatorios. ${ }^{5}$ Si la FCC falla en proteger significativamente la neutralidad en la red, se estarían violando los términos del BATS (Manner y Hernández, 20I4: 73).

El BATS integra claros principios de no discriminación en sus principales obligaciones. Engloba servicios de conmutación de paquetes, incluyendo servicios de banda ancha, los cuales Estados Unidos «expresamente incluyó [en el acuerdo] para proteger su creciente base de proveedores de servicio basados en IP» (Manner y Hernández, 20I4:

4. United State Trade Representative, USTR, 20I4 Trade Policy Agenda and 20I 3 Annual Report, anexo III. Disponible en <http://I.usa.gov/IRMPWHA>. El Documento de Referencia de BATS que Estados Unidos incluyó en su calendario de compromisos —que son legamente vinculantes para los países miembros de la $\mathrm{OMC}$ - establece un esquema regulatorio para las telecomunicaciones. Calendario de Compromisos de Estados Unidos GATS/SC/9o/Suppl.2. Disponible en <http://bit.ly/ıUXHfbd>.

5. Organización Mundial del Comercio, Servicios de Telecomunicaciones: Documento de Referencia: Grupo de negociación sobre telecomunicaciones básicas, del 24 de abril de 1996 (en adelante, Documento de Referencia OMC), artículos 2.I y 2.2 (a), disponible en <http://bit.ly/ınzidw7>. 
68). ${ }^{6}$ Los compromisos del BATS para servicios basados en el protocolo de internet incluyen varios principios clave que convergen con la neutralidad en la red, incluyendo transparencia, prácticas anticompetitivas, $\mathrm{y}$, sobre todo, interconexión justa (Manner y Hernández, 20I4: 60, 70). Bajo el BATS, la interconexión justa «quedará asegurada en cualquier punto técnicamente viable de la red» y debe ser proveída «bajo términos que no sean discriminatorios, [...] en una forma oportuna, [y] con tarifas basadas en el costo que sean transparentes y razonables, tengan en cuenta la viabilidad económica, y estén suficientemente desagregados para que el proveedor no deba pagar por componentes o instalaciones de la red que no necesite para el suministro del servicio». ${ }^{7}$

Lo que esto significa es que si la FCC hubiese mantenido las Reglas Propuestas de 2014, o adoptado reglas similares, permitiendo a los proveedores de banda ancha concertar un tratamiendo diferenciado al tráfico de internet, habría existido una contravención a las obligaciones legales de Estados Unidos bajo el GATS y el BATS de asegurar interconexión justa a los proveedores de servicios extranjeros. Tales estándares habrían permitido a los proveedores de banda ancha entablar negociaciones individuales con edge providers ${ }^{8}$ para crear carriles «rápidos» y «lentos» para el servicio de internet, ${ }^{9}$ los que por definición habrían

6. Con las siglas IP utilizadas en el texto citado se hace referencia a la expresión Internet Protocol, que es el protocolo para la comunicación de datos digitales. (N. de E.)

7. Documento de Referencia OMC, artículos 2.2 (a) y (b).

8. Se trata de los prestadores de servicios de internet que proveen de acceso a los clientes o usuarios finales. (N. del T.)

9. «Una persona que provee servicios de acceso a internet de banda ancha fija, en la medida en que esté contractualmente obligada, no deberá incurrir en prácticas comercialmente no razonables», Federal Communications Commission, Promoting and Protecting an Open Internet, GN Docket No. I4-28 (20I4) app. A $\int 8.7$ (20I4). Disponible en <http://fcc.us/IRWKJvo>. La FCC indicó que iba a determinar qué constituye una práctica comercialmente no razonable sobre una base de caso a caso, apoyándose en el estándar de la «totalidad de las circunstancias» (Sección i 16). La Corte de Apelaciones del Circuito del Distrito de Columbia (el «Circuito D.C.») indicó que el estándar de «razonabilidad comercial» otorga suficiente flexibilidad a los proveedores para que estos negocien acuerdos —incluyendo acuerdos de priorización pagada- en términos individuales. Cellco Partnership v FCC, 700 F.3d 534 (D.C. Cir. 20I2), confirma una orden de data roaming. Por tanto, la regla de prohibir las prácticas comerciales no razonables que regula a los proveedores de banda ancha, hubiese permitido a dichos proveedores 
violado el mandato de que las interconexiones sean proveídas en términos no discriminatorios, contenido en el BATS. ${ }^{\text {10 }}$ Adoptar dichas reglas hubiese dejado, por tanto, a Estados Unidos susceptible al riesgo de una denuncia ante la OMC por parte de otros Estados miembros de OMC a nombre de sus proveedores de servicios afectados por la medida. ${ }^{\text {I }}$

Del mismo modo, Estados Unidos se habría encontrado en una situación incómoda ante sus socios comerciales clave en América Latina y Europa. La neutralidad en la red es de particular importancia en Latinoamérica, donde aproximadamente el $85 \%$ de la población y el comercio de la región viven bajo un régimen legal que protege fuertemente este principio. En los últimos cinco años Paraguay, Chile, México, Colombia, Ecuador, Perú, Argentina y Brasil han adoptado legislación o regulación garantizando neutralidad en la red. Más aún, al menos un 90\% de todo el tráfico de internet latinoamericano pasa a través de Miami, y, por tanto, estaría sujeto a algún tipo de regulación por parte de Estados Unidos (Sparrow, 2013). Si la FCC no hubiese garantizado una potente protección de la neutralidad en la red, como lo hace la mayoría de los países de América Latina, el impacto comercial negativo habría sido severo (Cerda, 2014: 35-39).

Regulaciones como las propuestas por la FCC en mayo de 2014 podrían también afectar el comercio con Europa, otro continente que, en general, está adoptando potentes regulaciones para proteger la neutra-

entablar negociaciones individualizadas con edge providers a través de los cuales los proveedores habrían podido priorizar cierto contenido en perjuicio de otro, creando «carriles rápidos» para contenido priorizado y «carriles lentos» para todo el otro contenido.

Io. Mientras las Reglas Propuestas de 20I 4 de la FCC habrían, en principio, aplicado igualmente a todos los proveedores de servicio, domésticos y extranjeros, la decisión de dar prioridad a determinados agentes que pueden pagar por ese privilegio, sobre otros que no pueden, hubiese en efecto discriminado en contra de los últimos. Un tratamiento diferenciado de esas características del tráfico de internet habría tenido un efecto dispar y negativo en aquellos proveedores de servicio extranjeros que no están dispuestos o no son capaces de pagar la tarifa del "carril rápido", especialmente aquellos pertenecientes a países en vías de desarrollo. Para una discusión sobre la yuxtaposición entre asuntos de interconexión y neutralidad en la red, véase Ammori (2014).

I I. Estados Unidos es por lejos el miembro más litigioso del Órgano de Solución de Diferencias, y ha sido el querellante en 107 disputas y demandado en I2I. Organización Mundial del Comercio, «Diferencias por país/territorio". Disponible en <http://bit. ly/ıRsFEvR>. Véanse también (Heiskanen, I990: I 54-57) y (Barcelo, 2006: 36). 
lidad en la red. En abril de 20 I $_{4}$, el Parlamento Europeo aprobó la reforma de telecomunicaciones propuesta por la Comisión Europea que permitirá la existiencia de un único mercado digital (Yun Chee, 20I4), incluyendo salvaguardas para garantizar la neutralidad en la red y reglas estrictas que eviten bloquear o degradar los servicios de internet (Solon, 20I4). Holanda, Eslovenia, el Reino Unido y Noruega, entre otros, han consagrado protecciones sustantivas a la neutralidad en la red a través de legislación o regulación (MacDonald y Cannella, 20I4: 47), y Francia y Alemania están actualmente considerando legislar en el mismo sentido (MacDonald y Cannella, 20I4: 47; Marsden, 20I4: 83-84). ${ }^{\text {I2 }}$

Finalmente, la FCC escogió seguir las exhortaciones del presidente Obama y adoptó en su Open Internet Order de 2015 un marco para la regulación de internet en los Estados Unidos que es altamente protector de la neutralidad en la red en varios aspectos. Primero, la FCC definió el alcance de sus nuevas reglas aplicándolas «tanto a los servicios de acceso a internet de banda ancha móvil como fija». ${ }^{\mathrm{3} 3}$ Segundo, la FCC puso en efecto tres bright-line rules ${ }^{\mathrm{I} 4}$ que van al corazón de la protección de la neutralidad en la red: la prohibición del bloqueo, ${ }^{15}$ de la degración ${ }^{16}$ y de la priorización pagada. ${ }^{17}$ Finalmente, la FCC diseñó una forma de

I 2. Véase también RT, «Merkel challenges net neutrality by urging fast lanes for 'special services'» (20I4), disponible en <http://bit.ly/rOoYrV9>.

I3. Open Internet Order (2015), parágrafo 25.

I4. Un bright-line rule es una regla o estándar claramente definido que ayuda a resolver asuntos ambiguos utilizando estándares básicos que clarifican la ambigüedad y establecen una respuesta inequívoca. (N. del T.)

I 5 . Open Internet Order (2OI 5), parágrafo I I 2: «Una persona que provee servicios de acceso a internet de banda ancha, en la medida en que esté contractualmente obligada, no podrá bloquear contenido, aplicaciones, servicios o dispositivos inocuos que sean legales, sujeto a una administración razonable de la red».

I6. Open Internet Order (20 I 5), parágrafo I I9: «Una persona que provee servicios de acceso a Internet de banda ancha, en la medida en que esté contractualmente obligada, no deberá perjudicar o degradar el tráfico ilegítimo de internet sobre la base de contenido, aplicación, o servicio o uso de un dispositivo inocuo, sujeto a una administración razonable de la red».

I7. Open Internet Order (2015), parágrafo I 25: «Una persona que provee servicios de acceso a internet de banda ancha, en la medida en que esté contractualmente obligada, no deberá incurrir en priorización pagada. [...] La priorización pagada se refiere a la administración de la red del proveedor de banda ancha para directa o indirectamente 
alcanzar otros tipos de conducta que podrían no estar descritas por las bright-line rules al establecer su estándar de prohibir la «interferencia/ desventaja no razonable». ${ }^{8}$ Bajo esta regla, los ISP no pueden interferir o degradar no razonablemente, ni la capacidad de los usuarios finales de usar y acceder a servicios de banda ancha, ni la capacidad de los edge providers de poner a disposición de los usuarios finales dicho contenido. ${ }^{19}$

Entonces, ¿está Estados Unidos cumpliendo cabalmente todas las disposiciones antes citadas del GATS y el BATS? No completamente, debido a dos lagunas de cobertura creadas por la Open Internet Order de 2015 . En primer lugar, la FCC determinó que no iba a aplicar una bright-line rule a los servicios de datos auspiciados o planes zero rating, que debiesen estar derechamente prohibidos, y en su lugar los evaluará caso a caso bajo el estándar de prohibición a las «interferencias/desventajas no razonables». ${ }^{20}$ Zero rating generalmente se refiere a una práctica de las compañías de internet en que éstas pagan a ciertas empresas de telecomunicación para que ofrezcan a sus clientes acceso gratuito a través de su red móvil a los servicios en línea de la empresa auspiciadora, lo que se realiza a través de eximir el tráfico a los sitios de dichas compañías del tope de datos del usuario o permitiendo a los clientes sin un plan de datos acceder a dichos sitios (Center for Democracy and Technology, 20I4). Debido a que el zero rating se aparta de la neutralidad en la red -ya que a los datos auspiciados se les otorga prioridad sobre los no aus-

favorecer un tráfico sobre otro tráfico, incluyendo a través del uso de técnicas como traffic shaping, priorización, reserva de recursos, u otras formas de manejo preferencial de tráfico, sea: a) a cambio de una remuneración o beneficio de un tercero; o b) para beneficiar a una entidad afiliada».

I 8. Open Internet Order (2015), parágrafo I36: «Una persona que provee servicios de acceso a internet de banda ancha, en la medida en que esté contractualmente obligada, no deberá intervenir o desventajar de forma poco razonable: i) la capacidad de los usuarios finales de elegir, acceder y usar el servicio de internet de banda ancha o el contenido de internet, aplicaciones, servicios o dispositivos de su elección que sean legales; o ii) la capacidad de los edge providers para generar contenido, aplicaciones, servicios o dispositivos legales y ponerlos a disposición de los usuarios legales. La administración razonable de la red no será considerada una violación a esta regla».

I9. Open Internet Order (2015), parágrafo I 36.

20. Open Internet Order (20 I 5 ), parágrafo I 52. 
piciados-, la eventual aprobación de la FCC de algún plan zero rating podría resultar problemática por las razones expresadas anteriormente. En segundo lugar, la FCC dictaminó del mismo modo que ninguna de las bright-line rules o estándares relacionados con servicios de internet de banda ancha se aplicarían a los "acuerdos de intercambio de tráfico de internet», también conocidos como "interconexión». ${ }^{21}$ Interconexión se refiere a la interface de redes con otras redes en el intercambio de tráfico de internet. ${ }^{22}$ La FCC decidió que, "de momento», extender protecciones robustas a la neutralidad en la red a dichos acuerdos de intercambio «no se justificaba». ${ }^{23}$ En cambio, las disputas de interconexión serán revisadas caso a caso para evitar prácticas que puedan ser consideradas no razonables o injustas, ${ }^{24}$ un estándar amplio y vago (Gillula y Walsh, 2015). Como resultado, la puerta se mantiene abierta para acuerdos discriminatorios, como aquellos que involucran priorización pagada al interior de un acuerdo de interconexión, por ejemplo, entre ISP y redes de transmisión de contenido.

Cualquier disonancia en las reglas de neutralidad en la red entre Estados Unidos y algún socio comercial clave en América Latina y Europa podría preparar el escenario para posibles disputas en el futuro. Bajo las reglas de la OMC, «[una] disputa emerge cuando un país adopta políticas de comercio o toma alguna acción que uno o más miembros de la OMC considera que infringen los acuerdos de la OMC, o que no alcanzan a cumplir sus obligaciones». ${ }^{25}$ De hecho, Estados Unidos ha intercedido ante el Órgano de Solución de Diferencias (OSD) de la OMC a nombre de sus propios intereses de interconexión. En el año 2000, Estados Unidos realizó varias reclamaciones contra México por infracciones al Calendario de Compromisos de México, que incluían el Documento de Referencias del BATS. ${ }^{26}$ En 2004, un panel de la OMC concluyó que México había violado sus compromisos adoptados en el GATS al

2I. Open Internet Order (2015), parágrafo I94.

22. Open Internet Order (2015), parágrafo I94.

23. Open Internet Order (2015), parágrafo I95.

24. Open Internet Order (2015), parágrafos 202-205.

25. OMC, Solución de controversias. Una contribución excepcional. Disponible en $<$ http://bit.ly/IKjL6tp>.

26. OMC, Diferencia DS204. México. Medidas que afectan a los servicios de telecomunicaciones. Disponible en <http://bit.ly/206ZfmX>. 
no "asegurar interconexión a tarifas orientadas al costo", no "prevenir prácticas anticompetitivas por empresas que son grandes proveedores de telecomunicación» y no "asegurar acceso y uso no discriminatorio y razonable de las redes de telecomunicación». ${ }^{27}$ En respuesta al dictamen del panel OMC, México ha cumplido con el reporte del panel, dejando satisfechos tanto al OSD como a Estados Unidos.

En suma, varios factores bajo el GATS y el BATS pesaron en contra de las Reglas Propuestas de 2014 y en favor de implementar reglas potentes para proteger la neutralidad en la red, cumpliendo de esta forma sus obligaciones internacionales. Estados Unidos ha alcanzado dicho objetivo de forma exitosa, aunque aún insuficiente, a través de la adoptación de la Open Internet Order de 2015 . Estados Unidos no ha sido tímido a la hora de utilizar el Órgano de Solución de Diferencias de la OMC para empujar sus propios intereses en telecomunicaciones. De esta forma, sería difícil para ese país mostrarse sorprendido si otro Estado miembro lo demandase para impugnar las nuevas reglas de la FCC debido a que la Open Internet Order de 2015 parece autorizar los planes de zero rating bajo ciertas circunstancias, mientras expresamente excluye la interconexión del alcance de su protección a la neutralidad en la red. Estas lagunas en la Open Internet Order de 2015 respecto a la protección de la neutralidad en la red pueden eventualmente llevar a inconsistencias con las obligaciones de no discriminación de la OMC debido a servicios extranjeros basados en el protocolo de internet y requeridos por los tratados de comercio antes mencionados. ${ }^{28}$

27. OMC, Diferencia DS204. México. Medidas que afectan a los servicios de telecomunicaciones.

28. El artículo XIV del GATS permite distintas excepciones que reconocen el interés de los Estados parte para proteger la salud pública, el orden público, la seguridad, la moral, etcétera. Véase también Cottier y otros (2008), que describen el esquema legal de la OMC para las cláusulas de excepción general; Appellate Body Report, United States. Gambling, II 29I, WT/DS285/AB/R (27 de abril de 2005), confirma que la jurisprudencia de la OMC sobre el GAT relativa a excepciones es relevante para la interpretación del artículo XIV del GATS. Es importante hacer notar que estas excepciones son sustantivamente las mismas que las otorgadas por el artículo i9 (3) del PIDCP discutido anteriormente. Aunque Estados Unidos ha descansado en estas excepciones del GATS en oportunidades anteriores llevadas ante el OSD, estaría presionado para hacerlo acá por las mismas razones expresadas por la aplicación del artículo I9 del PIDCP. 


\section{CONSECUENCIAS RELACIONADAS CON DERECHOS HUMANOS DEL FRACASO DE LA FCC DE ADOPTAR REGLAS SUSTANTIVAS PARA PROTEGER LA NEUTRALIDAD EN LA RED}

Si la FCC hubiese implementado las Reglas Propuestas de 2014, u otras similares, dicha acción habría violado también las obligaciones internacionales relacionadas con derechos humanos de Estados Unidos de promover y proteger la libertad de expresión de una forma no discriminatoria. Permitir a los proveedores de banda ancha concertar un trato diferenciado o discriminatorio en el tráfico de internet habría infringido de forma inaceptable el derecho de todas las personas para buscar, recibir y difundir información, ideas y opiniones de forma igualitaria y en el medio de su elección. ${ }^{29}$ Como hicimos notar en la sección anterior, la FCC evitó este descalabro al adoptar varias bright-line rules en su Open Internet Order de 2015 para proteger la neutralidad en la red en servicios de banda ancha. Sin embargo, fracasó en extender estas protecciones a los acuerdos de interconexión, dejando la puerta abierta a potenciales abusos. De la misma forma, fracasó en prohibir el zero rating, una excepción per se a la neutralidad en la red. De esta forma, es posible argumentar que Estados Unidos se encuentra también vulnerable a litigación internacional en la arena de los derechos humanos.

Estados Unidos está obligado a respetar y proteger la libertad de expresión de forma no discriminatoria, inter alia, bajo el Pacto Internacional de Derechos Civiles y Políticos de las Naciones Unidas y la Declaración Americana de los Derechos y Deberes del Hombre (la Declaracion Americana)..$^{\circ}$ Tanto el Pacto Internacional como la Declaracion Ame-

29. Véase el artículo I9 de la Declaración Universidad de Derechos Humanos; el artículo I9 del Pacto Internacional de Derechos Civiles y Políticos; y el artículo 4 de la Declaración Americana de los Derechos y Deberes del Hombre.

30. Habiendo ratificado el Pacto Internacional, Estados Unidos es parte del tratado. Véase Consejo de Derechos Humanos, Recopilación preparada por la Oficina del Alto Comisionado para los Derechos Humanos: Estados Unidos de América, I2 de agosto de 2010 , A/HRC/WG.6/9/USA/2, disponible en <http://bit.ly/InziOy > $>$ La Declaración Americana de Derechos Humanos es vinculante a todos los Estados miembros de la Organización de los Estados Americanos en virtud de su membresía con esa organización y la ratificación de la Carta OEA, «Estado de firmas y ratificaciones», disponible en <http://bit.ly/IWigq5J>. Nunziato (2OI4: 3-4) describe el «compromiso de los Estados participantes de OSCE con la libertad de expresión... como está protegida en instrumentos internacionales». 
ricana consagran la libertad de expresión y la no discriminación como derechos fundamentales que los Estados deber proteger y promover. ${ }^{3 \mathrm{I}}$ La libertad de expresión es el derecho a buscar, recibir y difundir información, ideas y opiniones "a través de cualquier medio y sin consideración de fronteras»..$^{32}$ Ya se encuentra bien asentado el hecho de que este derecho se protege igualmente online que como se protege offline. ${ }^{33}$ En consecuencia, «el tratamiento de datos y tráfico de internet [no puede ser] basado en el dispositivo, contenido, autor, origen y/o la destinación del contenido, servicio o aplicación». ${ }^{34}$ Los Estados «deben tomar todas las medidas necesarias para fomentar la independencia [de internet] y asegurar el acceso de los individuos». ${ }^{35}$

La priorización pagada y otros acuerdos discriminatorios pueden actuar como restricciones inaceptables a la libertad de expresión al hacer el acceso a cierto tipo de contenido o redes más o menos difícil, dependiendo de si un individuo o compañía tiene la capacidad económica para acceder a esos acuerdos preferenciales. Sólo las compañías y organizaciones más acaudaladas son generalmente capaces de costear un tratamiento priorizado o preferencial para hacer su información y contenido más accesible a los usuarios (Strickler, 20I4). La capacidad para acceder a información —otro componente importante de la libertad de expresión - también puede verse afectada para aquellas personas o entidades que no pueden o eligen no pagar tarifas asociadas a la priorización o acceso preferencial a las redes o información.

3I. Artículos 2, I9 y 26 del Pacto Internacional; arts. 2 y 4 de la Declaración Americana. 32. Artículo I9 de la Declaración Universal de Derechos Humanos; artículo I 9 del Pacto Internacional; y artículo 4 de la Declaración Americana.

33. Resolución del Consejo de Derechos Humanos, $20{ }^{\circ}$ período de sesiones, «Promoción, protección y disfrute de los derechos humanos en internet», 29 de junio de 20I3, A/ HRC/20/L.I3, declara en el numeral I) que «los derechos de las personas también deben estar protegidos en internet, en particular la libertad de expresión, que es aplicable sin consideración de fronteras y por cualquier procedimiento que se elija». Disponible en $<$ http://bit.ly/Inq4Lmr>.

34. Declaración conjunta sobre la libertad de expresión e internet, de I de junio de 20II, parágrafo 5(a), disponible en <http://bit.ly/ıRsGuZv>.

35. Comité de Derechos Humanos, IO2. a sesión, Observación General 34: «Artículo I9. Libertad de opinión y libertad de expresión", de I 2 de septiembre de 20 I I, parágrafo I $_{5}, \mathrm{CCPR} / \mathrm{C} / \mathrm{GC} / 34$. Disponible en <http://bit.ly/rRsGuZv>. 
A pesar de que un Estado puede, bajo ciertas circunstancias, imponer ciertas restricciones a la libertad de expresión, sólo puede hacerlo si cumple con determinados criterios. Cualquier restricción propuesta debe ser: a) «otorgada por la ley»; ${ }^{36}$ b) para un fin legítimo como la seguridad nacional, el orden público, la salud pública y la moral; $; 7$ y c) proporcional, necesaria y «deberán estar relacionadas directamente con la necesidad específica de la que dependen»..$^{8}$ Aumentar las ganancias corporativas al proporcionar una ventaja competitiva a redes o proveedores de servicios con buenos recursos no es una causal legítima reconocida por el derecho internacional para restringir (relativamente) la libertad de expresión de los usuarios. Esto sugiere que tanto los planes de datos auspiciados como la priorización pagada al nivel de interconexión pueden resultar incompatibles con las reglas de libertad de expresión y no discriminación contenidas en el PIDCP y la Declaración Americana.

Cualquier insuficiencia en cumplir con sus obligaciones internacionales de derechos humanos podría exponer a Estados Unidos a denuncias por parte de los individuos perjudicados, organizaciones no gubernamentales e, incluso, otros gobiernos, al igual que en la arena del comercio. Por un lado, las denuncias pueden ser ventiladas en audiencias ante la Comisión Interamericana de Derechos Humanos, el órgano que monitorea el cumplimiento por parte de los Estados miembros de la Organización de Estados Americanos de los acuerdos regionales de derechos humanos como la Declaración Americana. Por el otro, defensores de los derechos humanos pueden denunciar la incapacidad de Estados Unidos de proteger la libertad de expresión solicitando una audiencia ante el Comité de Derechos Humanos de Naciones Unidas, la autoridad de Naciones Unidas que monitorea el cumplimiento de los Estados del Pacto Internacional. Como un Estado parte de ese tratado, Estados Unidos se encuentra sujeto a revisiones periódicas de su cumplimiento con el tratado por el Comité de Derechos Humanos, un órgano independiente

36. Artículo I9 (3) del Pacto Internacional.

37. Artículo ig (3) (a) y (b) del Pacto Internacional. Como ya se ha mencionado, estas excepciones son sustancialmente similares a las otorgadas por el GATS en su artículo XIV.

38. Comité de Derechos Humanos, parágrafo 22; ver también artículo I9 (3) del Pacto Internacional. 
compuestos por I 8 expertos internacionales. ${ }^{39}$ Tanto la Comisión Interamericana como el Comité de Derechos Humanos de Naciones Unidas publican sus dictámenes respecto del nivel cumplimiento de los Estados con sus respectivas obligaciones monitoreadas.

\section{LA REGULACIÓN DE LA FCC RESPECTO DE PROVEEDORES DE BANDA ANCHA COMO COMMONS CARRIERS SUJETOS A OBLIGACIONES DE NO DISCRIMINACIÓN BAJO LA LEY ESTADOUNIDENSE}

La Open Internet Order dictada por la FCC en marzo de 2015 marca un importante paso para corregir el error que la FCC cometió el año 2002, cuando rechazó clasificar a los proveedores de banda ancha como «servicios de telecomunicaciones» sujetos a obligaciones de common carriage bajo la Ley de Telecomunicaciones de I996. Al reclasificar a los proveedores de banda ancha como common carriers, la FCC armoniza el tratamiento de internet como un foro de expresión con el tratamiento histórico que Estados Unidos ha otorgado a otros foros de comunicación bajo la largamente reconocida doctrina del common carriage.

La doctrina del common carriage impone obligaciones sobre los conductos de expresión y comunicación masiva de propiedad privada consistentes en facilitar la expresión de otros individuos y prohíbe a dichos conductos el ejercer la discreción de determinar qué comunicaciones facilitar y cuáles censurar. Desde el comienzo de la era de las comunicaciones modernas en los años treinta, la FCC ha impuesto obligaciones sobre proveedores de comunicaciones interestatales (como compañías de teléfono o telégrafo) de facilitar la transmisión de todo contenido que sea legal. El Servicio Postal de Estados Unidos también ha sido regulado como un common carrier al cual le es requerido facilitar la transmisión de todo contenido legal y le es prohibido discriminar tal contenido (De Sola, I983: 7I-I07). Como Ithiel de Sola explica: «La ley del common carriage protege a los ciudadanos ordinarios en su derecho a comunicarse. Las reglas contra la discriminación están diseñadas para asegurar acceso a los medios de comunicación... Este elemento de la libertad civil es central a la ley del [common carriage]» (I983: 106).

El estatus de common carrier de los proveedores de comunicación

39. Comité de Derechos Humanos, documento disponible en <http://bit.ly/In9r8g9>. 
beneficia al público al otorgarle acceso a los conductos de comunicación bajo un principio de no discriminación. Como observó Jerome Barron, los individuos que dependen de common carriers para facilitar sus comunicaciones «se benefician del igualitarismo democrático que caracteriza al principio de acceso no discriminatorio asociado con la ley del common carrier» (Barron, I993: 37I).

El Congreso revisó la regulación de los proveedores de telecomunicación en la Ley de Comunicaciones de $1934,{ }^{40}$ la que otorgó a la recientemente creada FCC autoridad regulatoria sobre los proveedores de telecomunicaciones (compañías de teléfono y telégrafo), sin distinguir si éstas gozaban o no de poder monopólico, e impuso regulaciones de common carriege sobre dichos proveedores. ${ }^{4 \mathrm{I}}$ Bajo la Ley de 1934 , los common carriers se encontraron obligados a servir de conductos no discriminatorios para todo el contenido (legal) originado por otros. ${ }^{42}$

A lo largo de la mitad del siglo XX, el common carriage y las obligaciones de no discriminación fueron aplicados a conductos tradicionales como las compañías de teléfono. A comienzos de los años setenta, la FCC comenzó a considerar si imponer o no, y hasta qué punto, obligaciones de common carriage a procesos y servicios asistidos por computadoras. En una serie de Computer Inquiries, ${ }^{43}$ la FCC esencialmente creó dos categorías de servicios de comunicación asistidos por computadoras: los servicios básicos y los servicios mejorados. Los servicios «básicos» (más adelante, «telecomunicaciones»), como teléfonos y servicios de facsímiles, eran aquellos que ofrecían derechamente servicios de transmisión, los cuales fueron regulados como common carriers, sujetos a requisi-

40. 47 .C. \I5I (I934)

4I. Véase Am. Tel. \& Tel. Co. v U.S., (I936) 299 U.S. 232. Bajo la Ley de Comunicaciones de $\mathrm{I934}$, las obligaciones de common carriage eran impuestas sobre compañías que: I) incurrían en comunicación interestatal, 2) por cable, 3) por cualquier entidad que ofreciera sus servicios como un common carrier. La Ley definía common carrier en consideración a «si el carrier ofrece indiscriminadamente su servicio a todo tipo de personas» sin consideración a si la entidad gozaba o no de poder monopólico.

42. Sable Communications, Inc. v FCC (I989), 492 U.S. I I 5.

43. Las Computers Inquires fueron una secuencia de actos administrativos de la FCC realizados en ejercicio de sus funciones con el objeto de intentar regular los servicios de comunicaciones asistidos por computadores. (N. del T.) 
tos de no discriminación. ${ }^{44}$ Los servicios «mejorados» (más adelante, «servicios de información») eran aquellos en que se aplicaban procesos computacionales de procesamiento sobre la información del subscriptor, y los proveedores de dichos servicios fueron exceptuados de los requerimientos de common carriage y no discriminación.

En la tramitación de la Ley de Telecomunicaciones de I996, el Congreso volvió a revisar la categorización de los servicios sujetos a regulaciones de common carriage que fuera establecida en las Computer Inquiries. Bajo la Ley de I996, se dictaminó que los servicios de «telecomunicación» estarían sujetos a la regulación de common carriage (reemplazando la categoría de «servicios básicos»), mientras que los «servicios de información» fueron eximidos de la regulación de common carriage (reemplazando la categoría anterior, también exenta, de «servicios mejorados»).45 La Ley definía un «servicio de telecomunicación» como «el ofrecimiento de telecomunicaciones a cambio de una tarifa directamente al público... independientemente de las instalaciones utilizadas». ${ }^{46}$ El Ley mantenía obligaciones de common carrier sustantivas relativas a los proveedores de «servicios de telecomunicación», mientras dejaba a los proveedores de «servicios de información» sujetos a mucho menos regulación. Mientras la ley creaba una presunción que los telecommunications carriers serán tratados como common carriers, al mismo tiempo autorizaba a la FCC a abstenerse de aplicar cualquier disposición de la ley si la FCC determinaba que dicha aplicación era innecesaria para proteger en contra de la discriminación, para asegurar un servicio justo y razonable, para salvaguardar a los consumidores, o servir al interés

44. Computer Inquiry II, Final Decision, 77 FCC2d 384, parágrafo 96, 47 Rad. Reg.2d (P \& F) 669 ( (980). Servicio básico es el ofrecimiento de «la sola capacidad de transmisión sobre un canal de comunicación que es virtualmente transparente en términos de su interacción con la información proporcionada por el cliente».

45. Policy and Rules Concerning the Interstate, Interexchange Marketplace, Report and Order, I6 FCCR. 74I8, parágrafo 2 n.6 Comm. Reg. (P \& F) 64I (200I). «La Comisión ha concluido que el Congreso buscó mantener la distinción básica/mejorada en su definición de «servicios de telecomunicación» y «servicios de información» y que «servicios mejoras» e «servicios de información» deberían ser interpretados como cumpliendo la misma función».

46. Ley de Comunicaciones de I934, sección 3(44), enmendado y codificado en 47 U.S.C.A., sección I 53(44) (West, I99I). 
público. ${ }^{47}$ El Título II de la Ley de Comunicaciones establece un régimen regulatorio complejo impuesto sobre los common carriers, pero su deber esencial es el de no discriminar en el ofrecimiento de sus servicios, y en particular, no discriminar en contra de ciertos tipos de contenido a la hora de servir como conductos para la transmisión de dicho contenido.

En la «Consulta pública sobre acceso de alta velocidad a internet a través de cable u otros medios» $4^{8}$ del año 2002, la FCC concluyó erróneamente que el servicio de cable a través de módem era un «servicio de información» $\sin$ «un ofrecimiento separado de servicios de telecomunicación",49 en circunstancias que esta última hubiese hecho que dicho servicio estuviese sujeto a obligaciones de common carriage. La FCC dictaminó que la provisión de servicio de banda ancha de cable no contenía un servicio de telecomunicaciones separado porque la transmisión de los datos era una «parte esencial» de dicho servicio e integral a sus capacidades. ${ }^{\circ}$ Como un «servicio de información» sin un "ofrecimiento separado de servicio de telecomunicaciones», la provisión de internet banda ancha por parte de los operadores de cable fue eximido de las regulaciones de common carrier del Título II de la Ley de Comunicaciones. ${ }^{5 \mathrm{I}}$

Esta decisión errada resultó significativa ya que dio marcha atrás a la forma en que históricamente la FCC había regulado los servicios de telecomunicación. A lo largo de los años setenta y ochenta, la FCC formuló e implementó una distinción factible entre, por un lado, redes de transmisión común subyacentes, $y$, por otro, los servicios e información puestos a disposición a través de esa red. La mencionada Consulta Pública de 2002 derrumbó esa distinción crucial, y por primera vez permitió a conductos de comunicación discriminar contenido que debían transmitir a través de sus redes.

El principal paso en falso de la FCC al eximir a los proveedores de banda ancha de las obligaciones de common carriage y no discriminación

47. Véase National Cable \& Telecommunications Association et al. $v$ Brand X Internet Services et al., 545 U.S. 967, 975-76.

48. I7 FCCR. 4798 (2002).

49. I7 FCCR. 4798 (2002).

50. I7 FCCR. 4798 (2002).

5I. I7 FCCR. 4798 (2002). 
(luego aprobado por la Corte Suprema en National Cable \& Telecommunications Association et al. v. Brand X Internet Services et al.) fue su determinación de que los operadores de cable que proveían servicios de internet de banda ancha no estaban —en todo o en parte- ofreciendo «servicios de telecomunicaciones» $\mathrm{y}$, por tanto, no eran sujetos a obligaciones de common carriers (Nunziato, 2009: I I 5-I33). La FCC erró al rehusarse a reconocer que los proveedores de banda ancha ofrecen principalmente «servicios de telecomunicaciones» al público y sirven como conductos para la transmisión de la información de los usuarios, incluso si también ofrecen algún «servicio de información» adicional. Debido a que no logró reconocer la función de servicio de telecomunicaciones ofrecido por los proveedores de banda ancha, la FCC erróneamente removió las obligaciones de common carriage y no discriminación a que estaban sujetos los proveedores de banda ancha, dando pie atrás a cerca de un siglo de historia que encarnaba el «igualitarismo democrático que caracteriza los principios de acceso no discriminatorio asociado a la ley de common carrier» (Barron, I993: 37I).

$\mathrm{Al}$ regular a los proveedores de banda ancha, el Congreso y la FCC deberían guiarse por los principios subyacentes al derecho de las comunicaciones modernas. Estos indican que las democracias liberales requieren una ciudadanía bien informada, la que a su vez requiere que los ciudadanos disfruten la libertad de comunicarse y acceder a conductos de comunicación que operen en términos no discriminatorios. Los mismos principios que justifican regular a los operadores de teléfono y telégrafo y al servicio postal como common carriers sujetos a requerimientos de no discriminación — para proteger «a los ciudadanos ordinarios en su derecho a comunicarse»- son igualmente válidos al ser aplicados a los proveedores de banda ancha y de comunicaciones de internet (De Sola, I983: 106).

Las Reglas Propuestas de 2014 , que habrían permitido a los proveedores de banda ancha discriminar en contra de cualquier contenido o aplicación que estimasen por cualquier motivo que escogiesen, eran históricamente inconsistentes con el principio democrático igualitarista de proteger a los individuos en su libertad para comunicarse. Afortunadamente, la Open Internet Order de 2015 corrigió este error y requirió que los proveedores de banda ancha asumieran, como mínimo, las obligaciones de no discriminación que históricamente se han impuesto 
sobre los common carriers: el deber de facilitar y transmitir de forma no discriminatoria cualquier contenido que sea legal.

\section{CONCLUSIÓN}

En marzo de 2015 la FCC adoptó una serie de reglas sustantivas para proteger la neutralidad en la red, prohibiendo a los proveedores de servicio de banda ancha acordar un tratamiento diferenciado al tráfico de internet. ${ }^{52}$ Confiamos que, como parte de su análisis y justificación, la FCC haya considerado las consecuencias internacionales de sus acciones, junto con las domésticas, al implementar reglas que prohíben el trato diferenciado de tráfico de internet basado en el estatus económico del creador del contenido o del usuario final.

La FCC alcanzó su meta y evitó en gran medida, mas no toda, la exposición a objeciones internacionales ante la OMC y organismos internacionales de derechos humanos, al reclasificar a los proveedores de banda ancha como common carriers sujetos a obligaciones de no discriminación bajo a la ley estadounidense. La doble cuestión respecto al zero rating y la interconexión como posibles amenazas a una neutralidad en la red robusta aún no han sido debidamente abordadas en la Open Internet Order de 2015, dejando la puerta abierta para posibles disputas futuras. Sin embargo, lo que es seguro es que al adoptar estas nuevas reglas, la FCC avanzó pasos sustanciales hacia asegurar un cumplimiento cabal de las obligaciones internacionales comerciales y de derechos humanos de Estados Unidos en lo concerniente a la no discriminación y la libertad de expresión.

\section{REFERENCIAS}

Ammori, Marvin (20I4). "Interconnection disputes are network neutrality issues (of Netflix, Comcast, and the FCC)». CircleID, $7 \mathrm{de}$ abril de 20I4. Disponible en <http://bit.ly/ISoWQCw>.

BArCelo, John (2006). "The status of WTO rules in U.S. Law». Cornell Law Faculty Publishingns, Paper (36): I-34. Disponible en $<\mathrm{http}$ ://bit. ly/ ${ }_{\mathrm{I}} \mathrm{OoZ}{ }_{7} \mathrm{Kj}>$.

52. Open Internet Order (2005). 
BARron, Jerome (I993) «The telco, the common carrier model, and The First Amendment. The «Dial-A-Porn» precedent»». Rutgers Computer \& Technology Law Journal, I9 (I993): 37 I-386.

Center for Democracy and Technology (2013). "The importance of internet neutrality to protecting human rights online». Disponible en <http://bit.ly/IZs65 Bl>.

-. (20I4). «No. 208 net neutrality, zero-rating \& development: What's the data?», Internet Governance Forum. Disponible en <http://bit. ly/rOxweJz>.

Cerda, Alberto (20I4). «Net neutrality around the world panel». George Washington University Conference on Net Neutrality and Global Internet Freedom, 23 de octubre de 20I4. Disponible en <http://bit. ly/IZs6hzi>.

CotTier, Thomas y otros (2008). Article XIV GATS: General exceptions, in Max Planck commentaries on world trade law, WTO. Trade in Services, Rudiger Wolfrum et al. (eds.).

De Sola, Ithiel (1983). Technologies of freedom. Cambridge: Harvard University Press.

Gillula, Jeremy y Kit Walsh (20I5). "The FCC is keeping an eye on interconnection, but more clarity is needed», Electronic Frontier Foundation. Disponible en <http://bit.ly/ıKjMiwP>.

Heiskanen, Veijo (I990). "The relation of international and municipal law». Finnish Year Book of International Law, I: I 54-I 57.

Open InTERnet Order (2OI5). «Rules protecting and promoting the open internet, 80 Fed. Reg. I9.738». Disponible en <http://I.usa. gov/In 3 jPGa>.

MacDonald, Reagan y Giusy Cannella (2014). «Net neutrality: Ending network discrimination in Europa». En Luca Beli y Primavera de Filippi (eds.), The value of network neutrality for the internet of tomorrow (46-6o). Disponible en <http://bit.ly/rliMh $5 \mathrm{~V}>$.

Manner, Jennifer y Alejandro Hernández (20I4). "An overlooked basis of jurisdiction for net neutrality: The World Trade Organization Agreement on Basic Telecommunications Services». CommLaw Conspectus, 22 (I): 57-73. Disponible en <http://bit.ly/IUXJTho>.

Marsden, Christopher T. (20I4). Net neutrality: Past policy, present proposals, future regulation? En Luca Beli y Primavera de Filippi 
(eds.), The value of network neutrality for the internet of tomorrow (76-89). Disponible en <http://bit.ly/IliMh 5 V>.

Nunziato, Dawn (2009). Virtual freedom: Net neutrality and free speech in the internet age. Chicago: Standford Law Books.

-. (20I4). «The U.S. Federal Communications Commission's proposed rulemaking in the matter of protecting and promoting the open internet». Disponible en <http://bit.ly/IQ 5 RIQv>.

Solon, Olivia (20I4). "Victory for net neutrality in European Parliament». Wired. Disponible en <http://bit.ly/ıPy4 OnI >.

Somer, Jeff (2015). "What the net neutrality rules say». The New York Times, I 2 de marzo 2015. Disponible en <http://nyti.ms/Inq68lg $>$.

Sparrow, Thomas (2013). "Behind the scenes of Latin America's 'Internet Brain». BBC, 30 de enero de 20I3. Disponible en <http://bbc. in/IQdOXhW>.

STRICKLER, Yancy (2OI4). «FCC's 'Fast Lane' internet plan threatens free exchange of ideas». The Washington Post, 4 de julio de 2014. Disponible en <http://wapo.st/236XGYp >.

Yun Chee, Foo (20I4). «EU Parliament votes to end roaming, protect net neutrality». Reuters, 3 de abril de 20 I 4 . Disponible en $<\mathrm{http}: / / \mathrm{bit}$. $\mathrm{ly} / \mathrm{IU}_{2} \mathrm{O}_{3} \mathrm{Ec}>$.

\section{SOBRE LOS AUTORES}

Arturo J. Carrillo es profesor de enseñanza clínica del Derecho y Director de la Clínica de Derechos Humans en The George Washington University Law School. Además, es codirector de Global Internet Freedom and Human Rights Project de la misma universidad y es miembro del directorio de Global Network Initiative. Su correo electrónico es <acarrillo@law.gwu.edu> y su dirección postal es 2000 H Street NW, WDC 20052 , USA.

Dawn Carla Nunziato es profesora de Derecho en The George Washington University y es codirectora de Global Internet Freedom \& Human Rights Project de la misma universidad. Es autora de Virtual Freedom: Net Neutrality and Free Speech in the Internet Age (Stanford University Press). Su correo electronico es <dnunziato@law.gwu.edu> y su dirección postal es $2000 \mathrm{H}$ Street NW, WDC 20052, USA. 
El presente artículo es una traducción del artículo «The Price of Paid Prioritization. The International and Domestic Consequences of the Failure to Protect Net Neutrality in the United States» publicado originalmente en Georgetown Journal of International Affairs (summer 201 5 ). Fue traducido por Pablo Agustín Viollier, con expresa autorización de los autores. 